\title{
LESTER EMBREe
}

\section{AMBIENTE, TECNOLOGÍA Y JUSTIFICACIÓN.}

\section{ANÁLISIS REFLEXIVOS.}

\author{
Bucharest, ZetA Books, 2010, 210 PP.
}

por María-Luz Pintos Peñaranda

Este libro, traducción de Environment, Technology, Justification. Reflective Analyses (Bucharest, Zeta Books, 2008), está publicado en castellano por la editorial rumana Zeta Books, en soporte electrónico, dentro de nueva Serie concebida como Workhshop Texts (Textos del Taller Fenomenológico). El autor del libro, Lester Embree, es, a la vez, el padre de la sugerente idea de esta Serie que él mismo inaugura con el libro que aquí presentamos.

Aunque el autor no lo especifica en el título ni en el subtítulo, es evidente que en este libro no se trata de hacer "análisis reflexivos" sin más, sino análisis reflexivos "fenomenológicos"; y esto en el sentido en el que él interpreta y sugiere que ha de ser un análisis fenomenológico que por tal se tenga, dentro del ejercicio de la actitud fenomenológica. De hecho, en cada capítulo el autor vuelve siempre sobre qué es fenomenología, cuáles son los distintos enfoques que puede tener una reflexión $y$, más en concreto, qué es un "análisis reflexivo" de carácter fenomenológico. Hace esto con el propósito manifiesto de esbozar un marco de referencia y un enfoque fenomenológico con respecto al tema a tratar en cada capítulo.

La factura de la mayoría de los capítulos es muy semejante. El autor plantea en ellos el significado del propio concepto al que está dedica- 
do temáticamente cada capítulo. Para ello, primero adopta la actitud teórica en la que se pasan por alto las cosas-en-cuanto-encontradas (los noemata) para, a continuación, cambiar de actitud poniendo la atención en los encuentros (en cuanto noesis) de los sujetos con las cosas-en-cuanto-encontradas (intencionalmente) - actitud que es la propia de un análisis de corte fenomenológico. El autor considera que, de este modo, "las cosas en cuestión se llegan a comprender con una articulación y claridad mayores" (151). Por tanto, la impresión que brinda esta composición es que el tema de cada capítulo sobre todo le sirve al autor para ejemplarizar con él lo que él entiende por análisis reflexivo fenomenológico: "la reflexión y el análisis de encuentros y de cosas-en-cuanto-encontradas para aquellos que quieren hacer fenomenología más que hablar sobre ella $y$, por ende, para aquellos que quieren entender la fenomenología de manera fenomenológica y quieren así confirmar, corregir y/o ampliar los informes fenomenológicos previos y posiblemente, incluso, atreverse a componer nuevos ensayos relativos a cosas no exploradas con anterioridad" (20).
Tal vez uno tiene ante este libro la misma impresión de inicial desconcierto que cuando se enfrenta por primera vez a Fenomenología de la percepción de Merleau-Ponty. Al igual que ocurre en el libro francés, la gran cantidad de ejemplos que Lester Embree aporta en su intento de modificar nuestra mirada y volverla fenomenológica puede no surtir efecto si nos quedamos en la superficie anecdótica de cada ejemplo en sí. Para evitar esta impresión es preciso tener muy en cuenta, como base de todo el libro, que, en realidad, el autor actúa en todo momento como un ferviente seguidor del empeño de Husserl por anteponer la "actitud personalista" -en la que todos los sujetos nos hallamos y de la que no podemos salir mientras hagamos nuestra vida cotidiana- a la "actitud naturalista". Si uno lee este libro en esta clave podrá comprobar cómo capítulo a capítulo, página a página, ejemplo a ejemplo, el autor hace un gran esfuerzo en mostrar que las cosas no son sin más cosas naturales y que la realidad no es sin más la realidad en general, sino que todo está siempre referido a la experiencia del sujeto concreto, en la que todo se da y sin la que nada podría darse. No hace falta, pues, una gran perspicacia 
para entender este libro de Embree como una prolongación -buscada y querida- de la actitud husserliana. Es el propio Hussserl el que en 1930 aclara en una carta dirigida a su discípulo Dorion Cairns - del que fue alumno nuestro autor en la New School- que sus textos no presentan resultados para ser aprendidos a modo de axiomas, sino que ofrecen los fundamentos básicos -las herramientas de trabajo- para que cada uno pueda hacer fenomenología por sí mismo y, así, solucionar los problemas por sí mismo -esto es, usando el método fenomenológico de analizar y describir (lo que Embree llama "análisis reflexivo": "la expresión 'análisis reflexivo' es mi intento por capturar concisamente lo que es la fenomenología considerada como un enfoque" (197). De este modo, no es extraño que Embree cite literalmente en este libro (180), cual si fuera su manifiesto guía, y como ya había hecho en otros textos anteriores, el famoso $\S$ 27 de Ideas I. Es esta experiencia del sujeto, para quien las cosas (objetos, animales no humanos, humanos y todo lo perteneciente a nuestro mundo en general) forman parte de un mundo práctico, cada una con su carácter de uso (para el sujeto concreto del caso) y con sus dife- rentes cualidades de valor (para el sujeto concreto del caso), la que destilan todos los ejemplos ofrecidos en este libro. La riqueza de los análisis -"modestos análisis reflexivos"(197) - de Embree en este libro radica en aplicar la perspectiva fenomenológico-husserliana a ejemplos muy diversos, como el propio Husserl llamaba a hacer, con el fin de asegurarse así sortear el desajuste de la "actitud naturalista" con lo que es el vivir normal de cada sujeto.

Los once capítulos que componen este libro tienen su fecha original de publicación en años anteriores -entre 1989 y 2007- al de esta edición; algunos de ellos incluso ya han sido traducidos y publicados en una o varias lenguas más que la original inglesa. Si bien el índice sólo permite ver como única división la que hay entre los once capítulos de los que se compone el libro y ninguna otra división, en mi opinión lo cierto es que, por su temática, los capítulos forman tres grupos en alguna medida diferenciados. De hecho, la primera parte del propio título del libro refleja este triple agrupamiento: ambiente, tecnología, justificación. 
El primer grupo reúne los cuatro capítulos primeros, en los que el autor aborda temáticas relacionadas con la naturaleza y el medio ambiente: 1. "La acción para la salud de los ecosistemas"; 2. "La constitución del vegetal"; 3. "Problemas en torno al valor de la naturaleza"; 4. "La fundamentación no mundana del medioambientalismo".

La temática del segundo grupo gira en torno a la cultura y a la tecnología: 5. "La constitución de la cultura básica"; 6. "La racionalidad de la tecnología científica"; 7. "Acción indirecta y tecnología"; 8. "El dónde y el cuándo de las apariciones"; 9. "Existencia intrínseca y extrínseca".

Los capítulos del tercer grupo cierran el libro y están referidos a la justificación: 10. "¿Cuándo el fin no justifica los medios?"; 11. "La tolerancia analizada reflexivamente".

Para entrever de forma general la orientación y el desarrollo del primer agrupamiento, me ceñiré a su primer capítulo. Este capítulo, acerca de "La acción para la salud de los ecosistemas" es muy aclarador: "expresa los resultados obtenidos mediante observación teorética reflexiva en torno al modo como puede justificarse fenomenológica- mente la acción destinada a fomentar la salud en un ecosistema" (27). El autor aporta, como ejemplo de que es posible una acción individual en este campo, su propia experiencia con los dos jardines de su casa en Florida y va detallando esta experiencia de tal manera que, ciertamente, uno se ve llevado a admitir que, queriendo (willing), es posible contribuir activamente a una mejor salud del entorno, restauradora, "tanto como sea posible, [de] una versión aproximada de la diversidad de organismos nativos originaria, estable y sustentable" (20). Lo que Embree intenta mostrar es que para llegar a ese willing y poner manos a la obra es para lo que es preciso efectuar un "análisis reflexivo" previo, de corte fenomenológico, es decir, un análisis capaz de diferenciar de modo abstracto los componentes que se dan en todo encuentro entre el sujeto y su medioambiente (por supuesto, entre uno mismo y su medioambiente). Este encuentro, que es siempre "intencional" - sujeto y objeto sólo son lo que son como partes de lo que en sí es una unidad originaria e irrompible de mútua correlación-, nos muestra a un sujeto que se pone en este encuentro desde una actitud que, a la vez que es suya propia, 
también es cultural. Que es cultural se comprueba por el hecho de que algunos de estos componentes tienen la forma de hábitos adquiridos, que son los que se procederá a distinguir analíticamente: hábitos de creencia con respeto a lo encontrado y a nosotros mismos; hábitos de valoración; hábitos con respecto a aquello que se persigue hacer $o$ conseguir. En este análisis se distinguen efectos de estos hábitos en el medio ambiente; y, por tanto, entre las causas de estos efectos, se distinguen las diversas creencias que de un modo $u$ otro sirven de fondo para causar unos y otros efectos, sean estos negativos, sean positivos o sean indiferentes. Es obvio que distinguir a nivel cognoscitivo las causas de ciertos efectos en el medio ambiente nos facilitará descubrir y distinguir qué valores -intrínsecos y extrínsecos- están interfiriendo en el encuentro entre el sujeto y el medioambiente, provocando la asunción de determinados fines $y$, por tanto, la de sus correspondientes medios. Y qué duda cabe que realizar este ejercicio de análisis reflexivo que consiste en descomponer o desconstruir de esta manera lo que es un encuentro intencional y ponernos ante la evidencia de unos efectos $u$ otros, permite justifi- car o no justificar una determinada actitud, con sus creencias (believing), con sus valoraciones (valuing) y con lo que en ella se persiga (willing). El resultado final es que dicho análisis y dicha justificación pueden favorecer en nosotros, tanto a nivel personal como colectivo, un cambio a favor de la salud de los ecosistemas y contribuir a fundamentar todavía más, desde la fenomenología, ciertos movimientos políticos como el ecologismo (environmentalism) (105). Otros de los análisis que entran en este primer grupo continúan en esta misma línea y plantean, además, cuestiones relativas al valor de la naturaleza viviente (para los sujetos) o, incluso, cuestiones en torno a la constitución de lo vegetal.

En el segundo grupo de capítulos, Embree comienza por el análisis de "la constitución de la cultura básica", entendiendo por ella no la cultura superior o de gente cultivada, ni tampoco la que va ligada a una determinada interpretación, sino la que "subyace al pensamiento de sentido común tanto como al pensamiento científico de todo tipo (107). El capítulo aporta categorías con las que abordar la problemática especializada en fenomenología de 
la cultura. Lo propio de nuestro vivir en este mundo es que los humanos nos encontramos continuamente unos con otros. De este encuentro surge la cultura básica, a la vez que la cultura básica es la situación de fondo desde $-y$ en- la que cada miembro de la cultura se constituye como sujeto humano. La cultura básica expresa la familiaridad de los sujetos con el mundo (Lebenswelt) con el que cada sujeto se encuentra desde el inicio de su vida; es decir, con un mundo que se compone de cosas culturales que lo son tal precisamente por estar envueltas en creencias, valores y usos. El encuentro de unos sujetos con otros nunca tiene lugar desde una asituacionalidad cultural, es decir, desde una especie de abstracción de toda implicación cultural, sino más bien todo lo contrario: en el encuentro entre los sujetos humanos cada sujeto efectúa su encuentro desde su pertenencia a un grupo étnico, a una generación, a un género, a una lengua, a una clase social, a un estatus social, etc. "Los seres humanos son miembros de diversos grupos culturales y tienen identidades en relación con miembros del grupo propio y de otros grupos" (123). Esta múltiple pertenencia conlleva objetos culturales básicos, tales co- mo "creencias", "valoraciones" y un "querer" actuar que, además de tener sus actuaciones activas conlleva la "experiencia" concreta que cada sujeto arrastra siempre consigo pasivamente. El análisis y la descripción noético-noemática de Embree nos pone, por tanto, ante la constitución activa, o en pasividad, de todos estos objetos culturales, siempre para tomarlos desde la perspectiva de que son los que aporta en sí mismo cada sujeto concreto, subjetivamente, en cada encuentro con otro u otros. iLa mirada fenomenológica no está interesada en los objetos culturales en sí mismos sino en el hecho de que estos son un componente que nunca falta y que actúa activa o pasivamente en cada encuentro de un sujeto concreto con otros porque los portan los propios sujetos de estos encuentros!

El siguiente capítulo, en el que Lester Embree trata sobre "la racionalidad de la tecnología científica", prolonga este análisis general sobre la cultura básica. El núcleo del desarrollo de este capítulo va dirigido a analizar la tecnología en cuanto uso de equipo tecnológico; es decir, de un equipo cuya existencia consiste en su "para qué" es usado por los sujetos o, lo que es lo mismo, en su función o uso por los sujetos. "El 
uso de equipo puede ser científico o no científico, mecánico, artesanal e incluso especializado" (147), dice, pero en todos ellos inevitablemente es un uso racional; o, como el autor lo expresa, en todos ellos hay racionalidad; y la hay en la medida en que el uso lleva siempre consigo una teleología, una finalidad, que es puesta por el sujeto que da ese uso a ese tal equipo. Este uso de la tecnología, simple o compleja, nunca será un uso en abstracto sino que siempre lo será -y esto es lo que el autor quiere hacernos ver- en relación a las creencias, a las valoraciones, al querer y a las experiencias propias de los sujetos usuarios o sujetos consumidores concretos. Como el propio autor indica, el análisis que tome en cuenta todos estos componentes implicados en el uso de tecnología sin duda puede contribuir a justificar un determinado uso o, por el contrario, a desenmascarar viejos hábitos poniendo en evidencia su no justificación.

En el siguiente capítulo Embree aborda lo que él llama "acción indirecta y tecnología". En este capítulo el autor se propone distinguir y describir diferentes tipos de la acción indirecta centrándose sobre todo en ese tipo que es "la tecnología", al igual que se propone distinguir $y$ describir diferentes aspectos que van involucrados en este tipo de acción y también distinguir y describir objetos, sujetos e intermediarios que intervienen en esta acción. La idea que hace de gozne de este análisis es que "el mundo práctico [que es en el que vive cada sujeto en su vivir diario] es un campo de acción, donde parte de ella es directa pero una buena proporción es indirecta, y la tecnología es una especie de acción indirecta" (161), es decir, un fenómeno práctico pero indirecto, ya que supone un uso de equipo (equipmental). En la vida humana, la acción directa (que es la que es producida directamente por los movimientos corporales) ocupa una parte relativamente pequeña en ella, mientras que la acción indirecta ocupa en ella una gran parte.

El capítulo "El dónde y el cuándo de las apariciones" es un ejemplo de análisis reflexivo centrado en la percepción. Análogamente al esquema de los análisis anteriores, Embree trabaja aquí el enfoque fenomenológico de la cosa-en-cuanto-vista-yoída y del campo espacio-temporal, y nos pone ante interesantes preguntas cuya respuesta tendrá el lector o lectora que atreverse a pensar prosiguiendo por su cuenta el 
análisis repleto de sugerencias apuntadas por Embree.

El asunto del siguiente capítulo es acerca de si no hay en las cosasen cuanto-encontradas por el sujeto creencias intrínsecas y extrínsecas, valores intrínsecos y extrínsecos, y un querer actuar conforme a una finalidad intrínseca y extrínseca. Embree hace discurrir su análisis a través del ejemplo de las rutas y los destinos.

En el análisis que abre el tercer grupo de capítulos, el autor trabaja las relaciones de fines y medios con respecto a la justificación de unos y otros. Siempre todo lo que un sujeto hace, ha hecho o planea hacer, es un medio al servicio de algún fin. El análisis embreeano muestra "que aquello que puede conectarse como fin $y$ medio puede ser evidenciado por separado y luego creído, justificadamente, valorado y tal vez incluso querido en función de sí mismo, es decir, intrínsecamente" (194).

En el análisis que cierra este grupo de capítulos $y$, a la vez, el libro, Embree describe qué es la tolerancia y cómo puede ésta ser justificada reflexivamente. La tolerancia es una actitud social y cultural: la cosa encontrada en la tolerancia es la actitud de otro o de otra, y ésta es siempre una actitud específica que "puede ser analizada en relación a su ser experienciada, creída, valorada y querida por aquel que la tolera" (207). La persona tolerante comporta "valoraciones intentivas de las cosas que, correlativamente, tienen valores" (201). Los ejemplos que el autor elige aquí muestran los diversos tipos de valores que entran en juego, fortaleciendo así el análisis descriptivo de la tolerancia. Según él, la tolerancia es una actitud práctico-volitiva: "la persona tolerante se abstiene de oponerse a las actitudes que desvalora" (203); pero se abstiene de ello $y$, por tanto, las tolera, porque en su actitud está interviniendo "una valoración dirigida hacia algo distinto a la actitud del otro desvalorada" (204). El autor, en la segunda parte de este análisis, intenta ofrecer un método de justificación de lo que se tolera que no va dirigida a su aplicación a la tolerancia en general sino a casos particulares -que son en los que los sujetos nos encontramos en nuestra vida cotidiana.

Después de todo el recorrido por el libro podemos concluir que los análisis en él reunidos despliegan una sugestiva reflexión fenomenológica que sin duda es también una excelente base de partida para otros muchos "modestos análisis reflexi- 
vos" de quienes sepamos escuchar el mensaje embreeano. 